\title{
Bitemporal visual field defects associated with anomalies of the optic discs
}

\author{
MALGOLM V. GRAHAM \\ University Hospital of Wales
}

AN D

GRAHAM J. WAKEFIELD

Bath Eye Infirmary

Bitemporal visual field defects are usually associated with lesions of the optic chiasm. The field loss may be caused by pressure, primary neoplasia, arachnoiditis, or demyelinating disease. While the findings of a bitemporal defect should always direct attention to these possibilities, a careful inspection of the optic disc may reveal an ocular cause for the field loss.

The purpose of this communication is to emphasize the connection between inferior conus (Fuchs's coloboma) of the optic disc and a temporal or altitudinal field defect. Usually the conus is associated with inversion of the optic disc so that the retinal vessels emerge from the nasal side rather than the temporal side. Frequently depigmentation of the fundus adjacent to the conus is found; this is sometimes best appreciated in a retinal photograph or with indirect ophthalmoscopy. Difficulties in diagnosis can arise as is shown in the following patient.

\section{Case report}

Patient 1, a woman aged 62 years, complained of slight deterioration of the visual acuity in the left eye over a period of about 2 months. The corrected visual acuity had been $6 / 6-1$ in the right eye and 6/9 part in the left 2 years before. Apart from mild rheumatoid arthritis she enjoyed good health.

\section{Examination}

The visual acuity in the right eye was $6 / 6-\mathrm{I}$, with $-2 \cdot 25 \mathrm{D}$ sph., $-3 \mathrm{D}$ cyl., axis $5^{\circ}$, and in the left $6 / 18+$, with $-3 \mathrm{D}$ sph., $-2 \mathrm{D}$ cyl., axis $180^{\circ}$.

The fundi showed bilateral Fuchs's coloboma with inversion of the optic disc. The vessels showed congenital tortuosity. The right retina was normal. The central vein pulsated freely at the disc. The left retina showed evidence of a venous stasis retinopathy with scattered haemorrhages at the posterior pole. The blood pressure was i 9o/Ioo (on methyl dopa). The carotid arteries were normal. 
Visual fields (Fig. I)

RIGHT : upper temporal defect to small stimulus. The defect extended slightly across the midline (Fig. I $a$ ).

LEFT : upper defect extending across the midline, seen only with small stimulus (Fig. $\mathrm{I} b$ ).

(a)

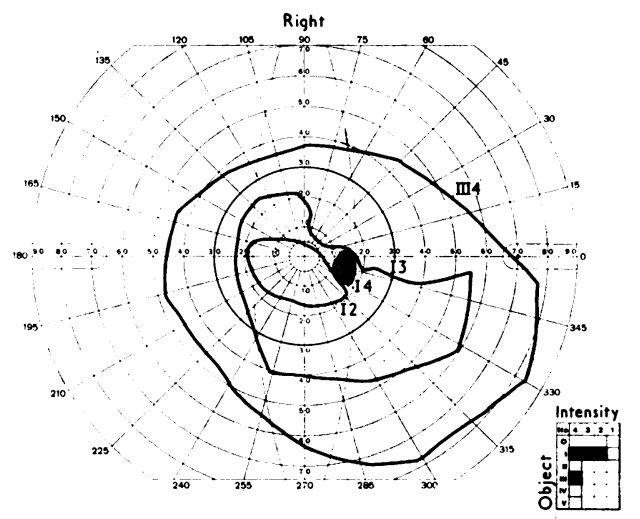

(b)

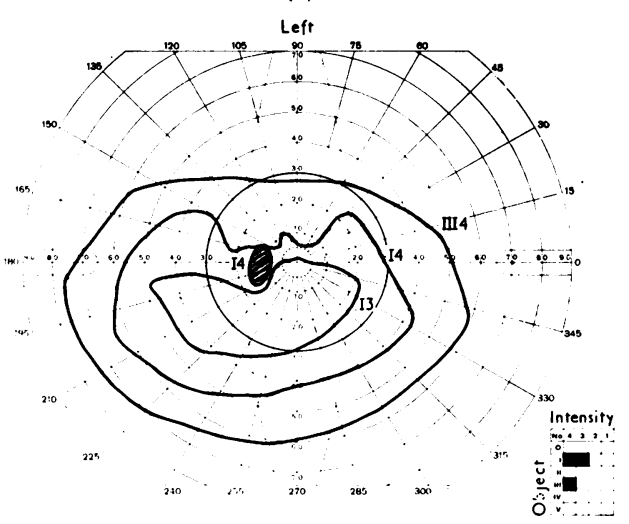

F I G. I $(a, b)$ Case I. Visual fields on November 6, 1969.

Radiography of the skull showed a pituitary fossa at the upper limit of normal size. An air encephalogram showed a doubtful shadow, suggesting a space-occupying lesion beneath both optic nerves anteriorly.

Although it was realized that the visual field defects could be accounted for by the optic disc abnormalities (Fig. I), the other findings suggested a dual pathology, and an exploratory craniotomy was carried out.

\section{Operation}

The chiasm was explored through a right frontal flap. There was extreme tortuosity of both carotid arteries which, with the ophthalmic arteries, had bulged upwards and medially to the optic nerve forming almost an aneurysmal dilatation in each case. The roof of the sella was normal. An attempt was made to separate adhesions between the optic nerve and the carotid artery on both sides to allow the nerve to move away to a limited extent if compression continued.

Result

The post-operative course was uneventful. The venous stasis retinopathy in the left eye cleared completely, and the visual acuity in the left eye improved to 6/9 part. The visual fields remained unaltered.

\section{Material and methods}

A series of sixteen patients with conus is summarized in the Table (overleaf, pp. 310 and $31 \mathrm{I}$ ). Two patients (Cases 5 and 8 ) had a normal disc in the second eye, and one (Case 9) had only a myopic crescent in the second eye. There were thirteen females and three males. Their age ranged between 7 and 72 years. The visual fields of both eyes were examined on the Goldmann kinetic perimeter, if one eye showed conus and/or inversion of the optic disc (Figs $2 a, b$ and $3 a, b$ ). Goldmann static perimetry was used on three eyes of two patients who showed field defects, a second curve being constructed using a more myopic correction when retinoscopy suggested an ectatic area of retina in the meridian of the field defect (Fig. 2c). Retinoscopy values tend to vary considerably in an eye with conus on examination slightly away from the fixation axis (Figs $2 d, e$ and $3 c, d$ ). 
(a)

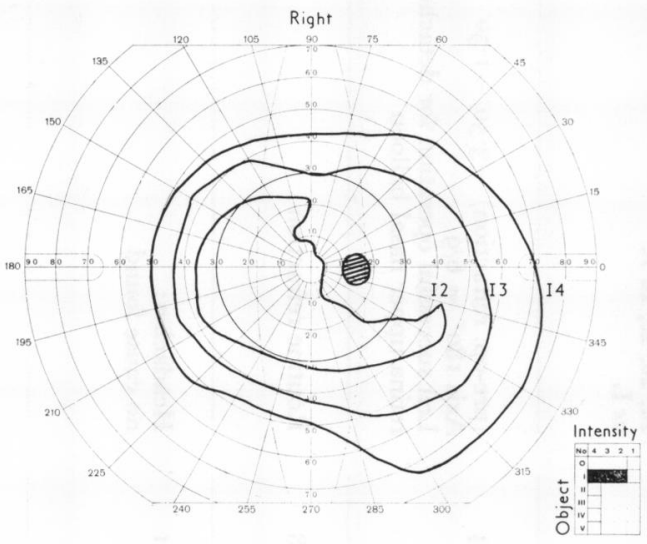

(b)

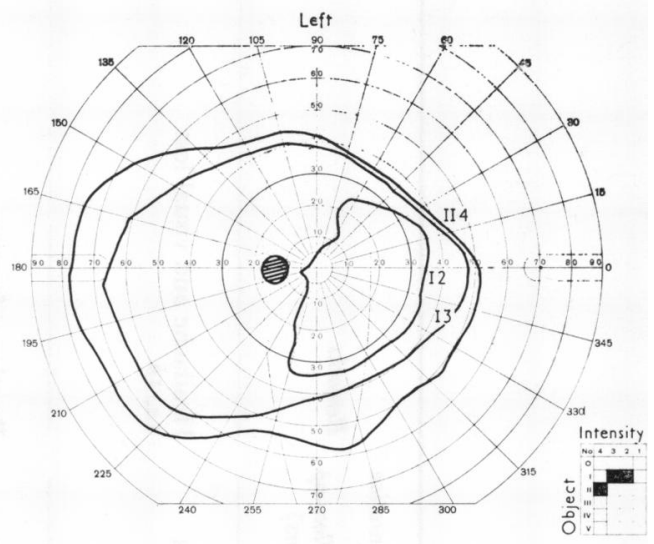

(c)

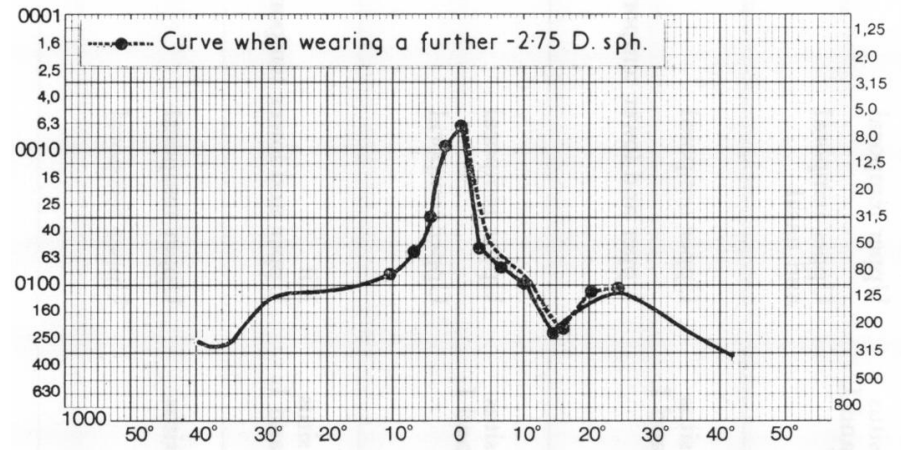

(d right)

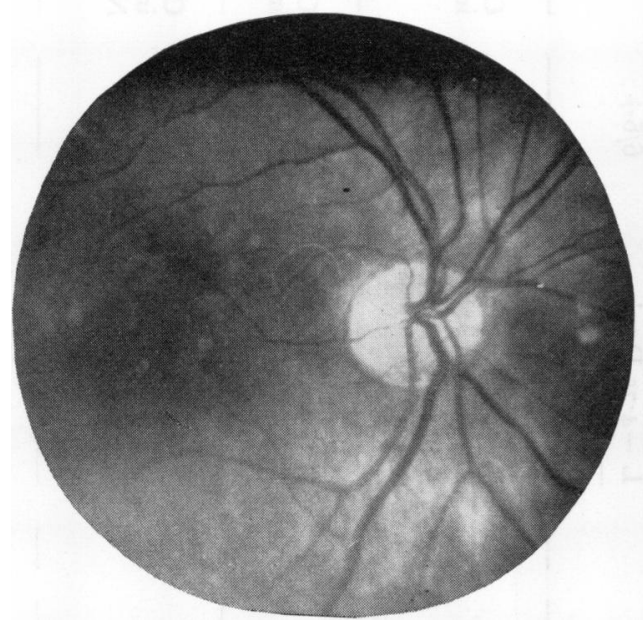

F I G. $2(a, b)$ Case 2. Visual fields on April 3, I97 I Retinal appearances, right and left. (e left)

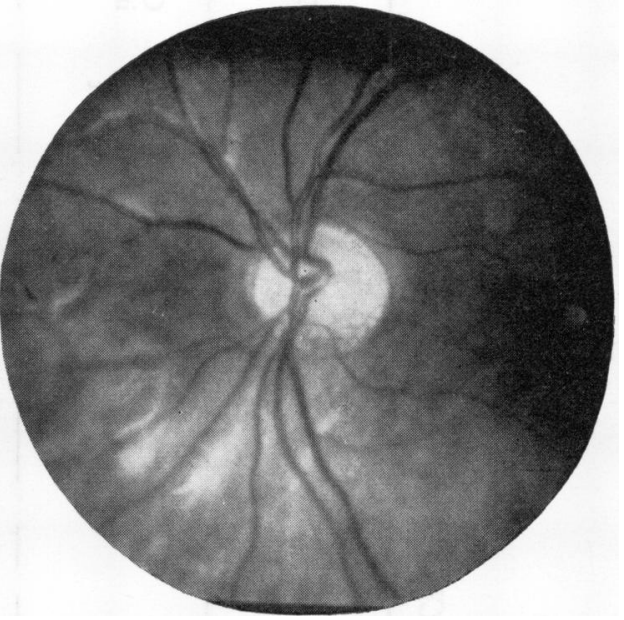

(c) Case 2. Static perimetry. (d,e) Case 2. 

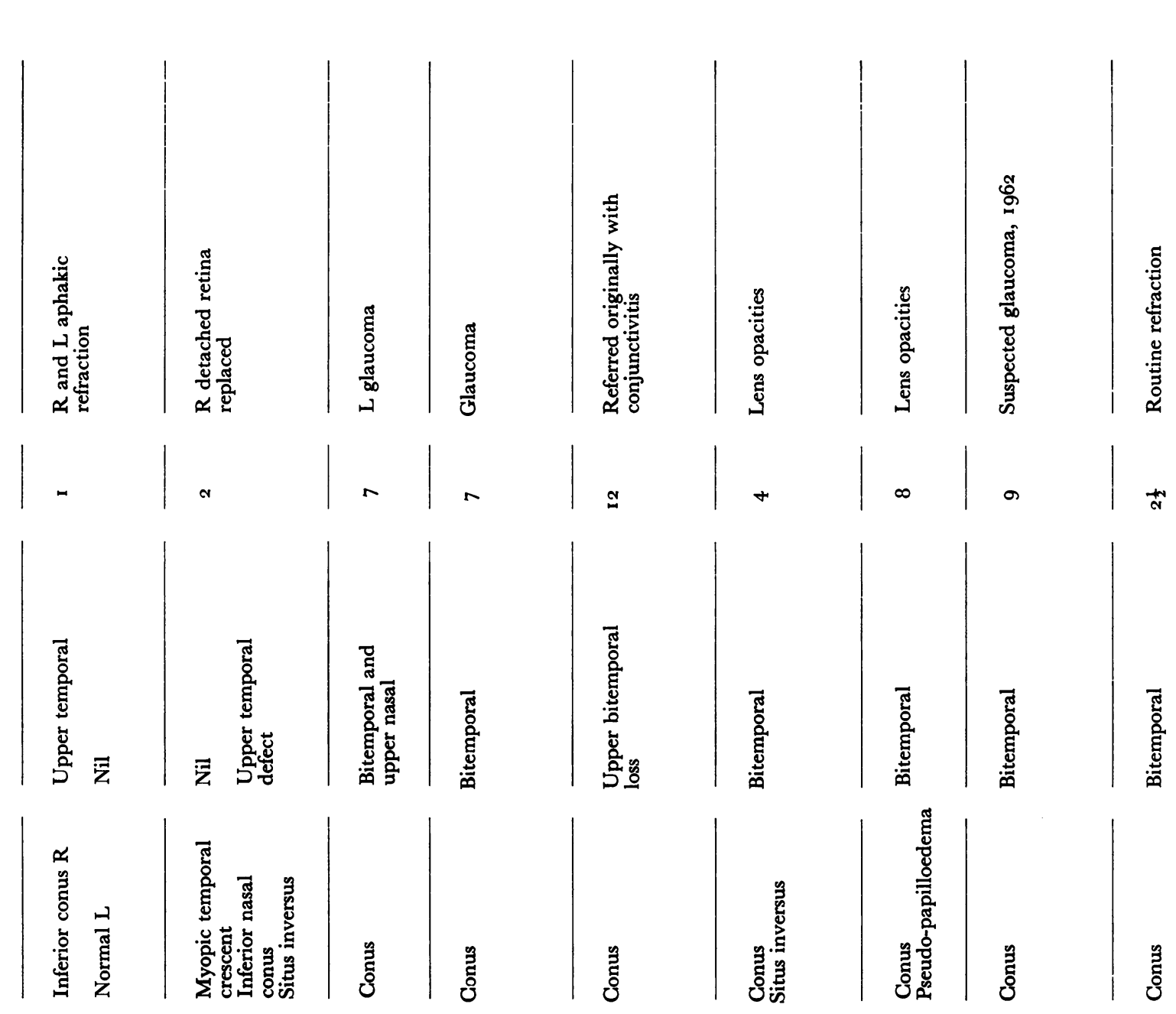

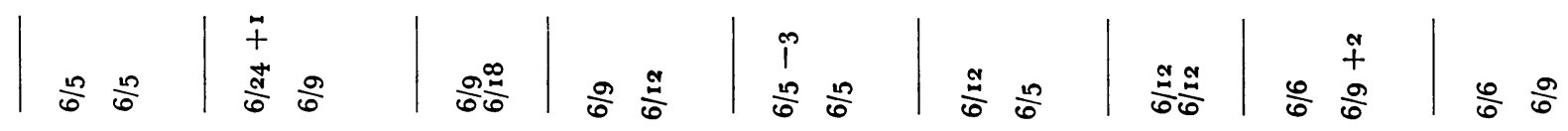
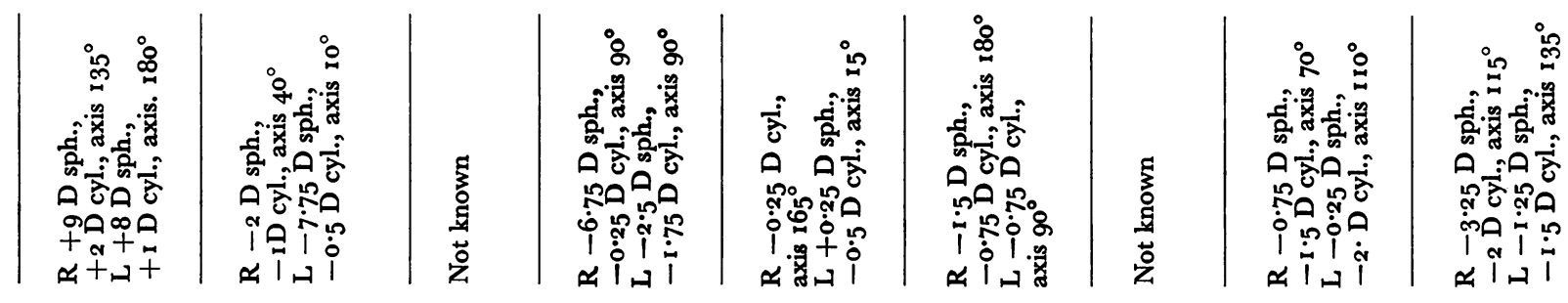

\begin{tabular}{l|l}
$\infty$ & $\infty$
\end{tabular}

$\approx \mid$ in $\mid$ \& 1

\& $|\therefore|$ in

己ֶํำ

ชี

1 is

$=1 z$

\begin{tabular}{l|l}
$\infty$ & 0
\end{tabular}

- $1=$

I क

$|\Sigma|$ is

in $\quad$ \% 

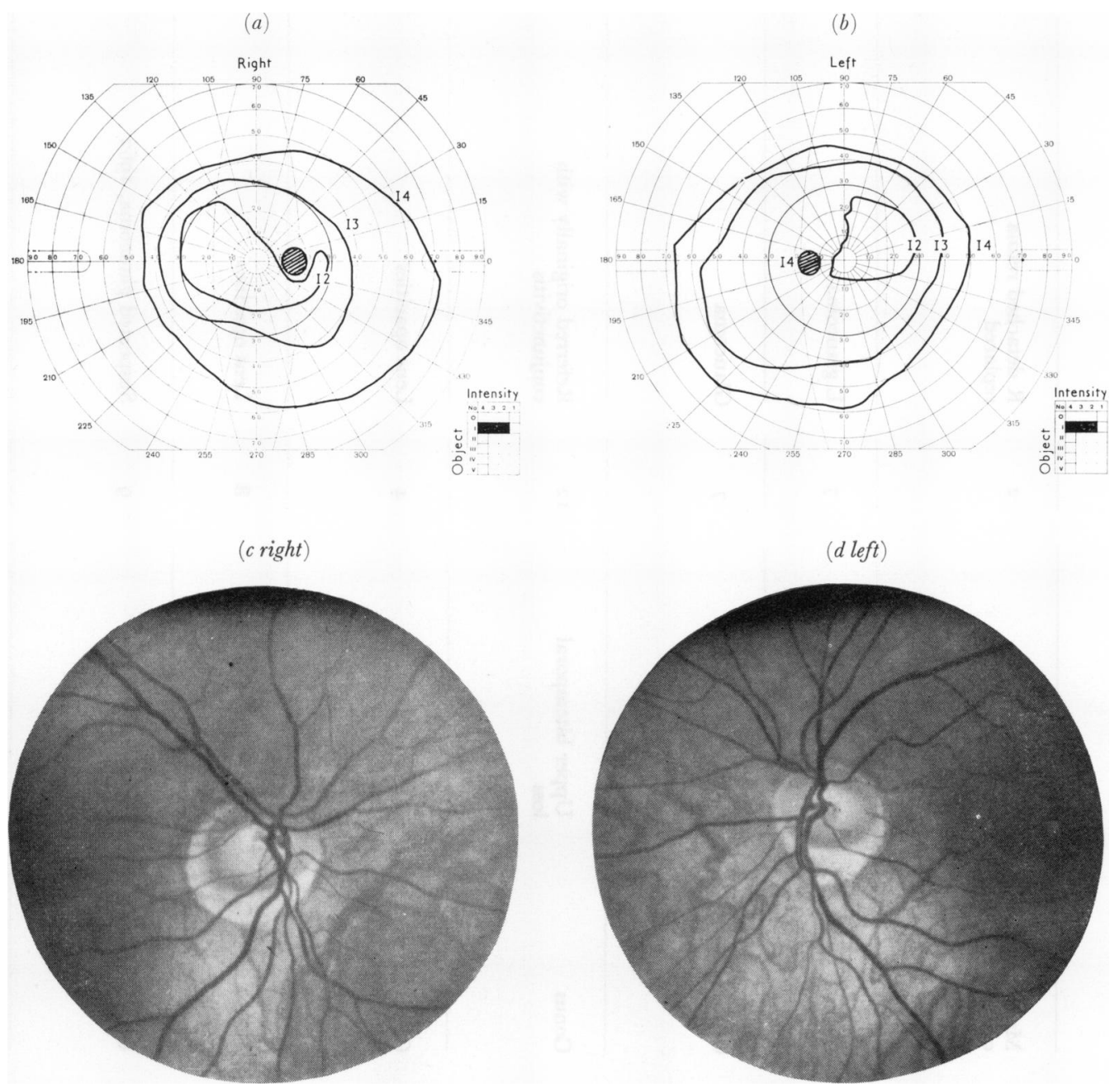

FIG. $3(a, b)$ Case 6. Visual fields on February 9, 197.$\quad(c, d)$ Case 6. Retinal appearances.

Two patients presented with retinal detachment, subsequently replaced: in one the affected eye did not show inversion or conus at the disc (Case 9); in the second the detached retina occurred in an eye with conus and inversion (Case 3 ). As the detachment in this case was an upper left nasal balloon, the field has been inclıded in the results. Other reasons for seeking medical advice were:

$\begin{array}{lll}\begin{array}{l}\text { Routine refraction } \\ \text { Glaucoma }\end{array} & 6 & \text { (Cases 2, 4, 6, 7, 8, I6) } \\ \text { Lens opacities } & 2 & \text { (Cases I0, I I) } \\ \text { Headache and visual loss } & \text { I } & \text { (Case I) } \\ \text { Suspected glaucoma } & \text { I } & \text { (Case I 5) } \\ \text { Headache } & \text { I } & \text { (Case 5) } \\ \text { Conjunctivitis } & \text { I } & \text { (Case I 2) }\end{array}$

Routine refraction 
In 28 out of 29 eyes which showed conus at the optic disc depression of the field was found to a small stimulus. In one patient the eye with a normal disc showed the characteristic upper temporal defect. Most of the defects were found in the upper temporal quadrant, but some extended to the lower quadrant or across the midline into the upper nasal quadrant.

Retinoscopy in the meridian of the defect at about $20^{\circ}$ to the optic axis invariably showed a slightly more myopic state than close to the fovea, suggestive of some degree of ectasia corresponding to the site of the field depression. Static perimetry across this meridian (Fig. 2c), with first the conventional correction and then with more myopic correction, only showed a very slight improvement in sensitivity. Peripheral fields were normal in all cases. All phakic eyes showing conus were associated with myopic astigmatism or compound myopic astigmatism.

Diminished retinal pigmentation was a marked feature in the lower nasal quadrants. This was well shown in Cases 3, 5, and 6, in which the fundus pallor extended close to the disc margins. The field defects in each case corresponded approximately to the pale area.

None of the patients was aware that there was any field defect present. No change in the field defect was noted in any case during follow-up periods which varied from I to 12 years.

\section{Discussion}

Szili ( 1883 ) showed that two phenomena were associated with inferior conus of the optic disc; the lower half of the fundus showed less pigmentation than the upper, and the error of refraction in the lower half was frequently greater than at the macula or temporal region. Rucker (1946) showed that temporal field defects occurred with situs inversus alone, and with conus alone. Fuchs ( 1882 ) emphasized the frequent association of situs inversus with inferior conus. Caccamise (1954), Berry ( 1963 ), and Odland (1967) described bitemporal field defects associated with inferior crescents, situs inversus, and thinning of the choroid. Schmidt (1955) showed that ectasias may give rise to field defects which disappear when higher myopic corrections are used. Maguire (197I) emphasized the value of static perimetry in this respect. That some degree of ectasia is present in the lower nasal quadrants of the retina in the present series is suggested by the higher myopic refraction in this area compared with the macula. All these cases were examined with correcting lenses. When higher minus lenses were used, a slight decrease of the field defect occurred, but this did not reach the normal level. The postulated cause of the field defects would seem, therefore, to be due to two possible factors:

(a) Some degree of ectasia of part of the globe. This is supported by the difference of the refraction in the affected area compared with that at the macula.

(b) Hypoplasia of the retina and choroid with fewer receptors per unit area. The decrease in pigmentation of the fundus in the lower nasal quadrants is advanced to support this theory.

In differentiating between bitemporal field defects due to ocular and chiasmal lesions, the behaviour of the field relative to the vertical meridian should be noted. Chiasmal lesions tend to respect the midline and end in a sharp edge, which in the early stages does not cross to the nasal side. Defects due to ocular lesions tend to slope obliquely across the midline and frequently involve part of the upper nasal quadrants.

\section{Summary}

Bitemporal visual field defects are described in a series of sixteen patients showing inferior conus of the optic disc with situs inversus. The nature of the field defect helps to distinguish an ocular from a chiasmal aetiology. The cause of the defect is postulated to be a combination of local retinal ectasia with hypoplasia. 


\section{References}

BERRY, H. (1963) Brit. 7. Ophthal., 47, 44I

CAccamise, w. c. (1954) Amer. 7. Ophthal., 38, 854

FUChS, E. (1882) v. Graefes Arch. Ophthal., 28, pt I, p. 139

maguire, c. (1971) Trans. ophthal. Soc. U.K., 91, 663

odland, м. (1967) Acta neurol. scand., 43,630

RUCKer, c. W. (1946) Arch. Ophthal. (Chicago), 35, 546

sChмid, т. (1955) Ophthalmologica (Basel), 129, 303

sZILI, A. (1883) Zbl. prakt. Augenheilk., 7, 358 